\title{
INTER-LIMB STEP ASYMMETRY IN CLOSED AND OPEN SKILL SPRINT ACCELERATIONS IN SOCCER PLAYERS
}

(1) University School of Physical Education in Wroclaw

original paper

DOI: https://doi.org/10.5114/hm.2021.98458

\author{
MAXIMILIAN M. WDOWSKI ${ }^{1}$, MARIANNE J.R. GITTOES ${ }^{2}$ \\ ${ }^{1}$ Faculty of Health and Life Sciences, Coventry University, Coventry, United Kingdom \\ ${ }^{2}$ Cardiff School of Sport \& Health Sciences, Cardiff Metropolitan University, Cardiff, United Kingdom
}

\begin{abstract}
Purpose. Inter-limb differences may decrease sports performance. There is a need to understand the comparison of asymmetries between open and closed skill tasks. The study investigated inter-limb step asymmetry responses to closed and open skill acceleration sprint running tasks.

Methods. 3D motion analysis kinematic data of step characteristics were collected from 20 male soccer players (mean \pm SD: $21 \pm 1.9$ years, $78.7 \pm 7.7 \mathrm{~kg}, 1.78 \pm 0.06 \mathrm{~m}$ ) for bilaterally located lower-limb markers during the initial $20 \mathrm{~m}$ of closed and open skill acceleration sprint running trials. Step velocity, step length, step frequency, flight time, contact time, and flight distance were calculated. In the closed skill conditions, players knew the final sprint distance before initiation. In the open skill conditions, players were informed of the final sprint distance (20 or $40 \mathrm{~m}$ ) immediately after run initiation.

Results. Significant asymmetries $(0.10-10.3 \%, p<0.05)$ in step characteristics were observed during closed skill acceleration sprint performances. Positive correlations $(r: 0.51-0.77, p<0.05)$ were evidenced between asymmetry scores in the closed skill and in the open skill 20-m and 40-m conditions. Asymmetry scores were not associated with sprint performance ( $r$ : from -0.13 to $0.30, p>0.05$ ), suggesting that asymmetry may be functional or dysfunctional.

Conclusions. Skilled soccer players can regulate step characteristic asymmetries across closed and open skill tasks. Symmetry should not be assumed when assessing open and closed skill acceleration sprint performance in soccer players. Practitioners should be aware of these asymmetries when implementing programmes.
\end{abstract}

Key words: soccer training, deterministic models, step characteristics, coaching, biomechanics, gait

\section{Introduction}

Inter-limb asymmetry compares the movement of one limb with respect to the other [1]. Evidence indicates that inter-limb differences measured across a range of tasks have a detrimental effect on physical and sports performance [2], and lead to potentially injurious consequences [3-5]. There has tended to be a stronger biomechanical research focus surrounding injury risk and occurrence when compared with physical or sports performance [2]. As a consequence of the increased interest in injury risk and occurrence, asymmetries of $<10 \%$ have been proposed as the target for when athletes are returning to sport [6]; both athlete and non-athlete populations who exhibit interlimb asymmetries in peak vertical countermovement jump force and the single leg hop test greater than 15\% have been associated with higher injury incidence than groups scoring below this threshold [3-5]. However, there is currently a lack of research examining the effect of asymmetries on sports performance [2].

Despite a large number of studies that have focused on kinematic or kinetic asymmetry in submaximal running and walking gait [7-10], asymmetry has rarely been investigated in sprint running. Sprint running is a key determinant of performance within soccer and many other sports [11, 12]. An explosive sprint was defined as the attainment of sprint speed from standing, walking, jogging, or running [13]. The majority of sprints in professional soccer are over short distances, with explosive sprints making up 23-30.5\% of these short sprints [13, 14], which makes the ability to acceler-

Correspondence address: Maximilian M. Wdowski, Science and Health Building, Faculty of Health and Life Sciences, Coventry University, Coventry, CV1 5FB, United Kingdom, e-mail: ac6071@coventry.ac.uk

Received: September 9, 2019

Accepted for publication: February 11, 2020

Citation: Wdowski MM, Gittoes MJR. Inter-limb step asymmetry in closed and open skill sprint accelerations in soccer players. Hum Mov. 2021;22(1):1-8; doi: https://doi.org/10.5114/hm.2021.98458. 
ate essential. According to the deterministic models of sprint performance by Hunter et al. [15], the player's velocity is determined by the step length (SL), step frequency (SF), and their sub-determinants. Therefore, adaptations in lower level variables, such as joint angles, would have an affect on higher level variables (e.g. contact time [CT], SL). Regardless of the importance of the ability to accelerate within soccer and other fieldbased sports, a current paucity of data examining acceleration sprint running asymmetries exists. Further research is required in a wide range of populations to more clearly determine if detrimental effects are shown in a variety of physical and sporting tasks and to examine if there are thresholds related to performance decrements [2].

Current research has investigated asymmetries during maximal sprinting tasks in youth athletes and reported weak relationships between the asymmetry variables and sprint velocity ( $r$ : from -0.24 to 0.39 , $p<0.05)$ [16]. With variables within the range of 2.3$12.6 \%$, the weak relationships reported by the respective study potentially indicate that sprint speed may not be detrimentally affected with the presence of interlimb asymmetry as high as $12 \%$ in a healthy youth population. However, Bishop et al. [2] highlighted that no specific details were provided on the sporting backgrounds of the participants. Meaning, any conclusions drawn from this study were not generalised to a homogenous, sporting sample of an equivalent or older age, such as adult soccer players. Therefore, further investigation into the sport-specific homogenous group is required.

Inter-limb kinetic and kinematic asymmetries have recently been assessed during the maximum velocity phase of a sprint in adult sprint-trained athletes [17] and found to be unrelated to mean sprint velocity. The respective study reported kinematic asymmetry values to be less than $10 \%$, step characteristics (step velocity [SV], SL, and SF) to be less than $2 \%$, and kinetic asymmetries to range from $0.1 \%$ to $93.2 \%$. However, with a low sample size of 8 , there is clarification required on the relationship between lower-limb asymmetries and sprint performance. Furthermore, closed skill protocols are typically used in studies investigating asymmetry, such as those by Exell et al. [17] and Meyers et al. [16], but for an application for sports that have an open skill element, there is a need to understand the comparison of asymmetries between open and closed skill tasks.

In a large variety of competitive soccer situations, the performer is unable to pre-plan a movement as a reactive physical and cognitive response is necessary.
The rate and extent of the biomechanical adaptation depend on the time required to physically and cognitively search for the appropriate movement pattern and the performer's experience within the particular constraints [18]. In soccer, and other field sports, the amendment of traditional closed skill sprint running protocols to include unanticipated changes in task constraint may be justified in order to better reflect an open skill environment [19, 20]. Currently, sprint step characteristic variables are investigated in closed skill environments. The existence of inter-limb asymmetry in acceleration sprint running and the extent to which inter-limb asymmetry is maintained or adjusted across sprint running tasks comprising differing constraints have yet to be fully elucidated. An explicit comparison of inter-limb asymmetry in open and closed skill tasks would potentially enable the development of closed skill screening methods that are representative of in-competition movement patterns in soccer. Closed skill screening protocols would help inform soccer players and coaches of potential imbalances and the effect of a player's asymmetry on performance.

The aim of this study was to empirically investigate inter-limb step asymmetry responses to closed and open skill acceleration sprint running tasks. Such information would provide insights into the degree of imbalance in step characteristics between limbs during acceleration sprint running in soccer players. It was hypothesised that significant inter-limb step asymmetries would be found in a number of the players, step characteristic asymmetries would be maintained across open and closed skill conditions, but there would be no correlation between performance and step characteristic asymmetries.

\section{Material and methods}

\section{Subjects}

Overall, 20 semi-professional male soccer players (mean \pm standard deviation of age: $21 \pm 1.9$ years, body mass: $78.7 \pm 7.7 \mathrm{~kg}$, and stature: $1.78 \pm 0.06$ $\mathrm{m})$ were recruited for the study. They were injury free and trained, played competitive soccer matches twice weekly, and further undertook 2 strength and conditioning sessions every week. The athletes were informed of the benefits and risks of the investigation.

\section{Procedures}

The study had a randomised repeated measures design. The players were required to attend 2 data col- 
lection testing sessions, a minimum of 48 hours apart, in the National Indoor Athletics Centre, Cardiff. Their whole-body mass and height were measured prior to the onset of the first data collection by using laboratory weighing scales (Avery Berkel Ltd, model ED01) and a stadiometer (Holtain Ltd). During each session, the athletes were required to perform 5 acceleration sprint running trials for a closed and 2 for open skill protocols such that a total of 10 player-specific trials were obtained for each condition across the 2 sessions. At the onset of the sprint and entering the 40-m sprint running zone, a set of visual stimulating Smartspeed ${ }^{\mathrm{TM}}$ light gates (Fusion Sport, Grabba International Pty Ltd, London) were illuminated at 2 different open skill sprint running target distances (20 m: O20, $40 \mathrm{~m}$ : O40) from the start of the $40-\mathrm{m}$ sprint running zone. On triggering the visual stimulating light gate, the competitors were instructed to accelerate maximally to the respective sprint running target distance. For the closed skill condition (C20), the participant was informed of the target distance $(20 \mathrm{~m})$ prior to the onset of the sprint running performance. The open and closed skill trials were implemented in a random order and each subject was given a minimum of 5-minute rest period between trials.

All sprint running performances were completed in a straight line on a 110-m indoor athletics track (National Indoor Athletics Centre, Cardiff). For each trial, the players were required to position themselves behind the start line in a standing position with the toes of their left foot immediately behind the start line, and the right foot placed comfortably behind. The athletes, who all wore rubber soled shoes, executed a 10-minute self-guided warm-up and familiarisation with the sprint running protocols. Preceding the warm-up, 2 active Cartesian Optoelectronic Dynamic Anthropometer (CODA) motion markers (Charnwood Dynamics Ltd, Leicester, UK) were attached to the individuals with adhesive double-sided tape. The markers were connected to marker drive boxes and located on a toe ( $5^{\text {th }}$ metatarsal-phalangeal joint) of the right side and a toe ( $1^{\text {st }}$ metatarsal head) of the left foot.

Four cx1 CODA motion scanners were horizontally aligned $3.0 \mathrm{~m}$ perpendicular to the plane of motion at $2.5 \mathrm{~m}, 7.5 \mathrm{~m}, 12.5 \mathrm{~m}$, and $17.5 \mathrm{~m}$ from the onset of the $20-\mathrm{m}$ sprint running zone. The respective scanners were used to capture (sample rate: $200 \mathrm{~Hz}$ ) 3-dimensional coordinate data of the active toe markers over the initial $20 \mathrm{~m}$ of each sprint condition. The CODA motion system was manually triggered prior to the initiation of the player's sprint running performance.

\section{Data processing and analysis}

The captured 3-dimensional coordinate data of each active marker were reduced to 2 dimensions ( $\mathrm{z}$ - vertical, y - anterior-posterior) and processed with a customised MATLAB program (2008b, The MathWorks, USA). Prior to further analysis, the respective raw coordinate data for the entire capture volume were filtered by using a fourth-order low pass Butterworth filter with a cut-off frequency of $12 \mathrm{~Hz}$, which was determined in a residual analysis.

Individual steps were defined within each running trial by using consecutive foot-ground touchdown events. For each step during the initial 20-m zone, touchdown (initiation of the stance phase) and toe-off (end of the stance phase) were determined with the time of the peak filtered z-acceleration [21] of the active toe markers and a minimum z-displacement. Forefoot striking was assumed for all conditions and step cycles. The method of calculating step characteristics was based on Salo et al. [22]. A step was subsequently defined from the instant of touchdown (initial) of one foot to the instant of the following touchdown (final) event for the contralateral foot [22]. The stance phase was defined as the instant of touchdown of one foot to the instant of the subsequent toe-off event for the same foot. Three outcome measures were calculated for all steps from the trials: SL was determined as the y-displacement of the active toe marker between the respective left and right foot touchdown events; SF was defined as the reciprocal of the duration between the initial and final touchdown events; SV was calculated as the product of SL and SF. Subcomponent measures, which were considered to mechanically underpin the outcome measures [15], were also obtained from the trials. The measures included the flight distance (FD), which was defined as the y-displacement of the active toe marker from toe-off of one foot to the touchdown of the contralateral foot; flight time (FT), which was defined as the period between the toe-off of one toe to the touchdown of the contralateral toe; and CT, which was determined as the period between the touchdown and initiation of stance phase to the toe-off and consequent ending of the stance phase for the same foot.

The mean and standard deviation of the step characteristics for both the right and left side were calculated for each trial and then data were pooled for each condition for every participant. The performance outcome velocity (VEL) was determined as the mean of the left and right side SV for a trial, and the mean \pm standard deviation was calculated for each individual in the closed skill 20-m condition. Asymmetry scores were 
subsequently established for every step characteristic for each participant $(n=20)$ in the separate conditions by using the method by Zifchock et al. [23].

\section{Statistical analysis}

The VEL, step characteristics, subcomponent measures, and asymmetry scores for the group and individual were tested for homogeneity of variance, sphericity, and normality with the use of the Levene's test, Mauchly's test, and Kolmogorov-Smirnov test, respectively. All players had a number of variables that were not normally distributed so non-parametric statistics were applied for further analysis. Differences between right and left side step characteristics for every participant in the C20 condition were quantified with the Wilcoxon signed-rank test (SPSS version 20.0, Chicago, USA), with the level of significance set at an alpha level of 0.05 . To assess differences in asymmetry scores between conditions at the group level, the repeated measures analysis of variance (RM ANOVA) with a Bonferroni post-hoc test for repeated measures was conducted. The level of significance was set at an alpha level of 0.01 . To ascertain a large effect with a statistical power of $85 \%$, a sample size of 20 was deemed appropriate ( $G^{*}$ Power 3.1.9.4, University of Kiel, Germany).

Spearman's correlation coefficients $(\rho)$ were calculated to investigate pairwise correlations between step characteristic asymmetry scores in each condition at the group level. Asymmetry scores of the step characteristics in the $\mathrm{C} 20$ condition were correlated with performance outcome (VEL) to explore the role of asymmetry in acceleration sprint performance. The level of significance was set at an alpha level of 0.05.

\section{Ethical approval}

The research related to human use has complied with all the relevant national regulations and institutional policies, has followed the tenets of the Declaration of Helsinki, and has been approved by Coventry University's Research Ethics Committee.

\section{Informed consent}

Informed consent has been obtained from all individuals included in this study.

\section{Results}

Table 1 provides the asymmetry scores for step characteristic variables in the closed skill 20-m (C20) condition for every participant. Overall, 16 of the 20 competitors displayed significant $(p<0.05)$ asymmetry in one or more of the step characteristics variables.
No significant differences $(p>0.01)$ were found between conditions for the asymmetry scores in any of the step characteristic variables (Table 2). The variable with the lowest asymmetry score was CT (C20: $1.00 \pm$ 0.96\%, O20: $1.10 \pm 0.93 \%$, O40: $1.04 \pm 0.98 \%$ ), whereas FT (C20: $4.23 \pm 3.00 \%$, O20: $5.81 \pm 2.81 \%$, O40: $4.98 \pm 2.55 \%$ ) was observed to have the largest asymmetry scores.

Table 1. Asymmetry scores (\%) for step characteristic variables in the closed skill $20-\mathrm{m}$ condition

\begin{tabular}{lllllll}
\hline Player & SV & SL & SF & FT & CT & FD \\
\hline 1 & 0.23 & $0.63^{*}$ & $0.41^{*}$ & $0.86^{*}$ & 0.00 & $0.65^{*}$ \\
2 & 0.12 & 2.23 & 1.91 & $4.03^{*}$ & 0.23 & 2.42 \\
3 & 0.68 & 0.79 & 0.02 & 0.37 & 1.4 & 0.83 \\
4 & 0.19 & 0.77 & 0.91 & 2.61 & 0.81 & 0.94 \\
5 & 0.10 & $2.92^{*}$ & $2.99^{*}$ & $5.65^{*}$ & 0.27 & $2.82^{*}$ \\
6 & $2.21^{*}$ & 1.93 & $4.08^{*}$ & $7.31^{*}$ & 1.31 & 1.83 \\
7 & $1.26^{*}$ & $2.04^{*}$ & $3.38^{*}$ & $6.10^{*}$ & 1.3 & 2.40 \\
8 & 0.75 & $1.01^{*}$ & 1.76 & 1.46 & 1.43 & 1.09 \\
9 & $1.52^{*}$ & 0.32 & $1.22^{*}$ & $2.26^{*}$ & 0.24 & 0.22 \\
10 & 0.21 & 0.34 & 0.28 & 0.00 & 0.00 & 0.24 \\
11 & $0.86^{*}$ & $3.29 *$ & $2.22^{*}$ & $5.32^{*}$ & 0.25 & $3.83^{*}$ \\
12 & $2.96^{*}$ & $1.84^{*}$ & $4.56^{*}$ & $10.3^{*}$ & 0.24 & $2.38^{*}$ \\
13 & $1.22^{*}$ & 0.02 & 1.16 & $3.38^{*}$ & 0.77 & 0.11 \\
14 & 0.97 & $1.83^{*}$ & $2.73^{*}$ & $6.77^{*}$ & 0.45 & $2.41^{*}$ \\
15 & $1.35^{*}$ & 1.12 & 2.13 & 4.28 & 0.53 & 1.14 \\
16 & $2.18^{*}$ & $1.12^{*}$ & $3.41^{*}$ & $5.29^{*}$ & $1.57^{*}$ & $1.43^{*}$ \\
17 & $2.73^{*}$ & $3.69^{*}$ & $6.44^{*}$ & $9.88^{*}$ & $3.14^{*}$ & $3.58^{*}$ \\
18 & 0.05 & $1.26^{*}$ & $1.05^{*}$ & $4.2^{*}$ & $2.00^{*}$ & $1.42^{*}$ \\
19 & $3.26^{*}$ & 0.98 & $4.37^{*}$ & $5.49^{*}$ & $3.38^{*}$ & 0.96 \\
20 & 0.13 & 0.33 & 0.36 & 0.00 & 0.75 & 0.29 \\
\hline
\end{tabular}

SV - step velocity, SL - step length, SF - step frequency, FT - flight time, CT - contact time, FD - flight distance * significant $(p<0.05)$ difference between right and left values $(n=10)$

Table 2. Mean \pm standard deviation of group $(n=20)$ step characteristics asymmetry scores (\%) in the closed skill 20-m (C20), open skill 20-m (O20), and open skill 40-m (O40) conditions

\begin{tabular}{cccc}
\hline Variable & C20 & O20 & O40 \\
\hline SV & $1.15 \pm 1.03$ & $1.13 \pm 1.03$ & $1.34 \pm 0.80$ \\
SL & $1.42 \pm 1.03$ & $2.27 \pm 1.17$ & $1.62 \pm 1.11$ \\
SF & $2.27 \pm 1.17$ & $2.70 \pm 1.66$ & $2.34 \pm 1.67$ \\
FT & $4.23 \pm 3.00$ & $5.81 \pm 2.81$ & $4.98 \pm 2.55$ \\
CT & $1.00 \pm 0.96$ & $1.10 \pm 0.93$ & $1.04 \pm 0.98$ \\
FD & $1.55 \pm 1.11$ & $2.22 \pm 1.16$ & $1.77 \pm 1.08$ \\
\hline
\end{tabular}

SV - step velocity, SL - step length, SF - step frequency, FT - flight time, CT - contact time, FD - flight distance There were no significant $(p<0.01)$ differences between conditions. 
Table 3. Spearman's correlations $(\rho)$ of the group $(n=20)$ step characteristic asymmetry scores between the closed skill 20-m condition (C20), the open skill 20-m (O20) and 40-m (O40) conditions, and the closed skill 20-m performance outcome step velocity (VEL)

\begin{tabular}{cccr}
\hline C20 & O20 & O40 & VEL \\
\hline SV & $0.77^{*}$ & $0.51^{*}$ & \\
SL & $0.60^{*}$ & $0.51^{*}$ & -0.13 \\
SF & $0.81^{*}$ & $0.86^{*}$ & 0.30 \\
FT & $0.71^{*}$ & $0.78^{*}$ & 0.22 \\
CT & $0.41^{*}$ & $0.60^{*}$ & 0.10 \\
FD & $0.60^{*}$ & $0.59^{*}$ & -0.06 \\
\hline
\end{tabular}

SV - step velocity, SL - step length, SF - step frequency, FT - flight time, CT - contact time, FD - flight distance * significant $(p<0.05)$ correlation with the C20 condition

Table 3 describes the pairwise correlations between condition responses for each of the step characteristic asymmetry variables. Each asymmetry score was either moderately or strongly correlated between conditions. For the SV asymmetry score, positive moderate-to-strong correlations were observed between the closed skill 20-m (C20) condition, and the open skill 20-m (O20) $(\rho=0.77, p<0.05)$ and open skill 40-m (O40) $(\rho=0.51, p<0.05)$ conditions. The SF asymmetry scores were strongly correlated between the closed skill 20-m (C20) condition, and the open skill 20-m (O20) $(\rho=0.81, p<0.05)$ and open skill 40-m (O40) $(\rho=0.86, p<0.05)$ conditions. The SL asymmetry scores were moderately correlated between the closed skill 20-m (C20) condition, and the open skill $20-\mathrm{m}(\mathrm{O} 20)(\rho=0.60, p<0.05)$ and open skill 40-m (O40) $(\rho=0.51, p<0.05)$ conditions. Step characteristic asymmetry scores were not significantly correlated with VEL.

\section{Discussion}

The aim of this study was to empirically investigate inter-limb step asymmetry responses to closed and open skill acceleration sprint running tasks in order to examine if asymmetries were transferred across open and closed skill acceleration sprint running tasks and to elucidate the effect of asymmetry on acceleration sprint performance. The main findings were that the majority of soccer players in the study displayed asymmetry in their step characteristics, and these asymmetries were maintained across closed and open skill acceleration sprint protocols. However, asymmetry in step characteristics was not associated with performance outcome.
Investigations are required into asymmetrical movement in a wide range of populations [23] to more clearly determine if detrimental effects are shown in sporting tasks and to examine if thresholds exist that are related to performance decrements [2]. The players in the current study displayed a low level of asymmetry across each of the step characteristics measured (from $1.00 \pm 0.96 \%$ to $4.23 \pm 3.00 \%$ ). Overall, 16 of the 20 athletes presented significant $(p<0.05)$ asymmetries in one or all of the step characteristics measured. In 10 competitors, significant asymmetries were observed in SV, indicating a consistently higher velocity in one leg than the other. According to the deterministic models of sprint performance [e.g. 15], the contribution of lower level step subcomponent asymmetry to overall SV asymmetry can be traced to FT, where 14 players displayed significant asymmetries. However, only 4 subjects had significant asymmetries in CT, suggesting that FT is the subcomponent that may be most susceptible to asymmetrical differences in soccer sprint acceleration. The inter-player differences in asymmetries reinforce the importance of individual analyses [22, 24]. These findings are important to practitioners as they highlight that there are often significant asymmetries present in soccer players' acceleration sprint running step characteristics and that individuals employ different mechanisms to ensure that performance is regulated.

It has been recently postulated that there may be relationships between a variety of asymmetry metrics, including step characteristics and sprint velocity [16], but the supporting literature is sparse. There is value in understanding whether asymmetry in performance is detrimental as it requires diverse conditioning responses to address limb dominance. The findings of the respective study supported the hypothesis that there would be no correlation between performance and step characteristic asymmetries. No relationships between VEL and step characteristics asymmetry scores were observed. These results are not unique to soccer players performing an acceleration sprint, as recent research investigating asymmetry in adult trained athletes during the maximum sprint phase [17] highlighted a lack of any relationship between step characteristics and performance outcome. The inconsistency between asymmetry and performance in acceleration sprint running suggested that asymmetry may be both functional and dysfunctional for different athletes. Exell et al. [17] argued that in performers that had an imbalance in strength or mobility around specific joints, asymmetry might be explained through the concepts of self-organisation [25] and be a functional require- 
ment to optimise performance. On the other hand, for other soccer players, asymmetry may be seen as noise and indicate that one side of the body is not performing as optimally as the other, requiring technique adjustment. Another explanation for no relationship between VEL and step characteristic asymmetry could be the low values of asymmetry present at the individual level (0.10-10.3\%). The majority of variables at the group and individual levels were below the $10-15 \%$ threshold that has been proposed as the target for when athletes are returning to sport [6], and reduced injury prevalence [3-5]. Current understanding has not yet extended thresholds to performance; however, our results suggest that no performance decrements exist in lowerlimb step characteristic asymmetries below the threshold of $10-15 \%$. Therefore, it is important for practitioners to understand that lower limb asymmetry may be ever present during soccer acceleration sprint performance but that it may only be detrimental to specific individuals.

The second hypothesis predicted that when compared with a planned (closed skill) sprint running task, a reactive (open skill) task would be consistent in the quantity of asymmetry in step characteristics and subcomponents across the different tasks. Traditional protocols utilised to assess and develop field sport performance have been criticised owing to the lack of unpredictability in the movement tasks employed [26]. This hypothesis was found to be true as there were no significant differences $(p>0.01)$ observed in asymmetry scores across conditions, as well as moderate-to-strong correlations ( $\rho$ : $0.41-0.84$ ) between asymmetry variables in each condition. Asymmetry in acceleration sprint running may therefore transfer across different open skill tasks. These findings support the concept of a strong self-organisation by the soccer players in adapting to unanticipated changes in task constraint [25]. Asymmetry in step characteristics and their subcomponents may thus be a learned response that is stable irrespective of task constraint in skilled performers. Such understanding may allow for the creation and implementation of performance screening tests quantifying asymmetry in a closed skill task that are still applicable to movements in an open skill competition environment.

It is important to mention that the approach used in the current study of assessing the step characteristic asymmetry during an acceleration sprint run can provide quick and accessible information over a large $20-\mathrm{m}$ capture volume within a field setting. The information gathered from such protocols can help inform the practitioner of underlying asymmetries in a performer, and through an individual level analysis determine whether the asymmetries are functional or dysfunctional. Further study is required that would examine the joint kinematics and kinetics during these acceleration sprint runs to broaden the understanding of the mechanisms that underpin, according to the deterministic models of sprint performance [e.g. 15], the asymmetry found at top level step characteristics and their subcomponents. A limitation of the current study is that we averaged all the steps over the 20-m sprint performance, which may have limited the sensitivity of detecting asymmetries. Investigation into the different phases of a sprint performance (e.g. early, mid, and late acceleration subphases [27]) may provide more sensitive information on the nature of lower-limb asymmetries and their relationship with performance.

From a data collection perspective, symmetry should not be assumed when assessing top level deterministic parameters of acceleration sprint performance in soccer players. Where only a unilateral 2-dimensional analysis is possible, caution should be used when inferring from results, and practitioners should be aware of potential differences in the limb not analysed. The results of our study suggest that lower-limb step characteristics asymmetries during acceleration sprint performances are very individual and that asymmetries may be functional or dysfunctional to performance, depending on the individual. Therefore, practitioners should assess sprint performance asymmetries at the individual level and, in line with current understanding [2], apply bilateral and unilateral strength, balance, and core training to reduce lower-limb asymmetries.

\section{Conclusions}

In conclusion, this research highlights that soccer players display significant asymmetries in their step characteristics during acceleration sprint performances in both open and closed skill tasks. No relationships between SV and step characteristics asymmetry scores were observed, providing evidence that asymmetry may be both functional and dysfunctional for different athletes. Finally, skilled soccer players are able to regulate step performance asymmetries across closed and open skill tasks, which provides a basis for the development of closed skill asymmetry performance screening protocols in soccer. Future research should examine whether these step characteristic asymmetries are present in the underlying mechanics of joint kinematics and kinetics. 


\section{Disclosure statement}

No author has any financial interest or received any financial benefit from this research.

\section{Conflict of interest}

The authors state no conflict of interest.

\section{References}

1. Keeley DW, Plummer HA, Oliver GD. Predicting asymmetrical lower extremity strength deficits in collegeaged men and women using common horizontal and vertical power field tests: a possible screening mechanism. J Strength Cond Res. 2011;25(6):1632-1637; doi: 10.1519/JSC.0b013e3181ddf690.

2. Bishop C, Turner A, Read P. Effects of inter-limb asymmetries on physical and sports performance: a systematic review. J Sports Sci. 2018;36(10):1135-1144; doi: 10.1080/02640414.2017.1361894.

3. Barber SD, Noyes FR, Mangine RE, McCloskey JW, Hartman W. Quantitative assessment of functional limitations in normal and anterior cruciate ligamentdeficient knees. Clin Orthop Relat Res. 1990;255:204214; doi: 10.1097/00003086-199006000-00028.

4. Grindem H, Logerstedt D, Eitzen I, Moksnes H, Axe MJ, Snyder-Mackler L, et al. Single-legged hop tests as predictors of self-reported knee function in nonoperatively treated individuals with anterior cruciate ligament injury. Am J Sports Med. 2011;39(11):2347-2354; doi: 10.1177/0363546511417085.

5. Impellizzeri FM, Rampinini E, Maffiuletti N, Marcora SM. A vertical jump force test for assessing bilateral strength asymmetry in athletes. Med Sci Sports Exerc. 2007;39(11):2044-2050; doi: 10.1249/mss.0b013e31 $814 \mathrm{fb} 55 \mathrm{c}$.

6. Kyritsis P, Bahr R, Landreau P, Miladi R, Witvrouw E. Likelihood of ACL graft rupture: not meeting six clinical discharge criteria before return to sport is associated with a four times greater risk of rupture. Br J Sports Med. 2016;50(15):946-951; doi: 10.1136/bjsports-2015 -095908 .

7. Hamill J, Bates BT, Knutzen KM. Ground reaction force symmetry during walking and running. Res Q Exercise Sport. 1984;55(3):289-293; doi: 10.1080/ 02701367.1984.10609367.

8. Vagenas G, Hoshizaki B. Functional asymmetries and lateral dominance in the lower limbs of distance runners. Int J Sport Biomech. 1991;7(4):311-329; doi: 10.1123/ ijsb.7.4.311.

9. Zifchock RA, Davis I, Hamill J. Kinetic asymmetry in female runners with and without retrospective tibial stress fractures. J Biomech. 2006;39(15):2792-2797; doi: 10.1016/j.jbiomech.2005.10.003.

10. Laroche DP, Cook SB, Mackala K. Strength asymmetry increases gait asymmetry and variability in older women. Med Sci Sports Exerc. 2012;44(11):2172-2181; doi: 10.1249/MSS.0b013e31825e1d31.
11. Lockie RG, Murphy AJ, Schultz AB, Jefferiess MD, Callaghan SJ. Influence of sprint acceleration stance kinetics on velocity and step kinematics in field sport athletes. J Strength Cond Res. 2013;27(9):2494-2503; doi: 10.1519/JSC.0b013e31827f5103.

12. Moir G, Sanders R, Button C, Glaister M. The effect of periodized resistance training on accelerative sprint performance. Sports Biomech. 2007;6(3):285-300; doi: 10.1080/14763140701489793.

13. Di Salvo V, Baron R, González-Haro C, Gormasz C, Pigozzi F, Bachl N. Sprinting analysis of elite soccer players during European Champions League and UEFA Cup matches. J Sports Sci. 2010;28(14):1489-1494; doi: 10.1080/02640414.2010.521166.

14. Di Salvo V, Gregson W, Atkinson G, Tordoff P, Drust B. Analysis of high intensity activity in Premier League soccer. Int J Sports Med. 2009;30(3):205-212; doi: 10.1055/s-0028-1105950.

15. Hunter JP, Marshall RN, McNair PJ. Interaction of step length and step rate during sprint running. Med Sci Sports Exerc. 2004;36(2):261-271; doi: 10.1249/01. MSS.0000113664.15777.53.

16. Meyers RW, Oliver JL, Hughes MG, Lloyd RS, Cronin JB. Asymmetry during maximal sprint performance in 11- to 16-year-old boys. Pediatr Exerc Sci. 2017;29(1): 94-102; doi: 10.1123/pes.2016-0018.

17. Exell T, Irwin G, Gittoes M, Kerwin D. Strength and performance asymmetry during maximal velocity sprint running. Scand J Med Sci Sports. 2017;27(11):12731282; doi: 10.1111/sms.12759.

18. Sanders R, Li S, Hamill J. Adjustment to change in familiar and unfamiliar task constraints. J Sports Sci. 2009;27(6):651-659; doi: 10.1080/02640410902729758.

19. Sheppard JM, Young WB. Agility literature review: classifications, training and testing. J Sports Sci. 2006; 24(9):919-932; doi: 10.1080/02640410500457109.

20. Araújo D, Davids K, Bennett SJ, Button C, Chapman G. Emergence of sport skills under constraints. In: Williams AM, Hodges NJ (eds.), Skill acquisition in sport: research, theory and practice. London: Routledge; 2004; 409-433.

21. Bezodis I, Thomson A, Gittoes M, Kerwin D. Identification of instants of touchdown and take-off in sprint running using an automatic motion analysis system. In: Menzel HJ, Chagas MH (eds.), Proceedings of the $25^{\text {th }}$ International Symposium on Biomechanics in Sports. Ouro Preto: International Society of Biomechanics in Sports; 2007; 501-504.

22. Salo AIT, Bezodis IN, Batterham AM, Kerwin DG. Elite sprinting: are athletes individually step-frequency or step-length reliant? Med Sci Sports Exerc. 2011; 43(6):1055-1062; doi: 10.1249/MSS.0b013e318201f6f8.

23. Zifchock RA, Davis I, Higginson J, Royer T. The symmetry angle: a novel, robust method of quantifying asymmetry. Gait Posture. 2008;27(4):622-627; doi: 10.1016/ j.gaitpost.2007.08.006. 


\section{HUMAN MOVEMENT}

M. Wdowski, M. Gittoes, Sprint acceleration inter-limb asymmetry

24. Zuzgina O, Wdowski MM. Asymmetry of dominant and non-dominant shoulders in university level men and women volleyball players. Hum Mov. 2019;20(4):1927; doi: 10.5114/hm.2019.85095.

25. Kugler PN, Turvey MT. Self-organization, flow fields, and information. Hum Mov Sci. 1988;7(2-4):97-129; doi: 10.1016/0167-9457(88)90009-7.

26. Sheppard JM, Young WB, Doyle TLA, Sheppard TA, Newton RU. An evaluation of a new test of reactive agility and its relationship to sprint speed and change of direction speed. J Sci Med Sport. 2006;9(4):342-349; doi: 10.1016/j.jsams.2006.05.019.

27. Bellon CR, DeWeese BH, Sato K, Clark KP, Stone MH. Defining the early, mid, and late subsections of sprint acceleration in Division I men's soccer players. J Strength Cond Res. 2019;33(4):1001-1006; doi: 10.1519/JSC. 0000000000003088. 